\title{
Sigma pole position and errors of a once and twice subtracted dispersive analysis of pi-pi scattering data
}

\author{
R. Kamiński ${ }^{\mathrm{a}}$, R. Garcia-Martin ${ }^{\mathrm{b}}$, P. Grynkiewicz ${ }^{\mathrm{c}}$, J. R. Peláez $^{b}$ \\ a Department of Theoretical Physics, H. Niewodniczański Institute of Nuclear Physics, Polish Academy \\ of Sciences, 31-342 Kraków, Poland \\ bepartamento de Física Teórica, II (Métodos Matemáticos), Facultad de Ciencias Físicas, Universidad \\ Complutense de Madrid, E-28040, Madrid, Spain
}

cul. Paulińska, 6/18 Kraków 31-065

\begin{abstract}
We show how the new precise data on kaon decays together with forward dispersion relations, sum rules and once- and twice-subtracted Roy's equations allow for a precise determination of the sigma meson pole position. We present a comparison and a study of the different sources of uncertainties when using either once- or twicesubtracted Roy's equations to analyze the data. Finally we present a preliminary determination of the $\sigma$ pole from the constrained dispersive data analysis.
\end{abstract}

\section{Introduction}

Roy's equations (RE), based on twicesubtracted dispersion relations and crossing symmetry conditions for $\pi \pi \rightarrow \pi \pi$ amplitudes were obtained in 1971 [1]. In recent years, these equations have been used either to obtain predictions for low energy $\pi \pi$ scattering, sometimes using Chiral Perturbation Theory (ChPT) 223], or to test ChPT [456, as well as to solve old data ambiguities [7]. Roy eqs. are relevant for the sigma pole, whose position has also been predicted very precisely with the help of ChPT [8].

Our group [5]6] has also used Roy eqs. with Forward Dispersion Relations (FDR) to obtain a precise determination of $\pi \pi$ scattering amplitudes from data consistent with analyticity, unitarity and crossing. On purpose, we have not included ChPT constraints, so that we can use our results as tests of the ChPT predictions. Unfortunately, the large experimental error of the scattering length $a_{0}^{2}$ of the isospin 2 scalar partial wave, becomes a very large error for the sigma pole determination using RE. For this reason, a new set of once-subtracted RE, called GKPY eqs. for brevity, have been derived. Both the RE and GKPY equations provide analytic extensions for the calculation of poles in the complex plane. Here we present preliminary results for the $\sigma$ pole in the S0 wave obtained from GKPY eqs. and analyse the different sources of errors comparing their size with those from RE.

\section{Roy versus GKPY Equations}

In practice Roy's equations relate real parts (output) of the $S 0, P$ and $S 2$ partial wave amplitudes $f_{\ell}^{I}(s)$ below some energy scale $s_{\max }$, with the imaginary parts of all other partial waves and high energy parametrizations (input). Both GKPY eqs. and RE can be split into three terms:

$$
R e f_{\ell}^{I}(s)=S T_{\ell}^{I}(s)+K T_{\ell}^{I}(s)+D T_{\ell}^{I}(s) .
$$

The "subtraction terms" $S T(s)$ are polynomials whose coefficients are linear combinations of the S0 and S2 scattering lengths. For standard, twice subtracted, Roy's eqs., $S T$ are first degree polynomials, thus growing quadratically with energy, whereas for GKPY they are just constants. Hence, the uncertainty due to the poor experimental knowledge of the isospin 2 scalar scattering length, $a_{0}^{2}$ becomes a very large source of error at high energies for standard Roy's eqs.

The "kernel terms" $K T(s)$ parametrize the contribution of the $S 0, P$ and $S 2$ waves $(\ell=0,1)$ 
up to a given energy $s_{\max }$, whereas the "driving terms" $D T(s)$ refer to all waves above $s_{\max }$ and also to $\ell>1$ partial waves from threshold. In our case $\sqrt{s_{\max }}=1420 \mathrm{MeV}$ and for higher energies we use Regge parametrizations. Both the kernel and driving terms are integrals of the kind:

$$
\sum_{I^{\prime}=0}^{2} \sum_{\ell} \int d s^{\prime} K_{\ell \ell^{\prime}}^{I I^{\prime}}\left(s, s^{\prime}\right) \operatorname{Im} f_{\ell^{\prime}}^{I^{\prime}}\left(s^{\prime}\right) \text {. }
$$

The integration kernels $K_{\ell \ell^{\prime}}^{I I^{\prime}}\left(s, s^{\prime}\right)$ are different for RE and GKPY eqs. The important fact being that, for high $s^{\prime}$, they decrease as $\sim 1 / s^{\prime 3}$ for RE, but as $\sim 1 / s^{\prime 2}$ for GKPY eqs. Hence GKPY eqs. are more sensitive to the high energy input.

By comparing Fig. 1 for RE with Fig. 2 for GKPY eqs. very remarkable differences (especially for the $S 0$ wave) can be observed in the relative sizes of the $S T$ and $K T$ terms. Please note the different scales. The curves represent the real part (output) of the amplitudes, i.e., $s^{1 / 2} \eta \sin \delta / 2 k$, where $\delta$ and $\eta$ are the phase shifts and elasticities for the preliminary amplitudes of the Constrained Data Fit described in the talk by J.R. Peláez in this conference. This fit describes data and has been constrained to satisfy FDR, RE. GKPY eqs. and some crossing sum rules.

Note that, for RE, the $S T$ and $K T$ terms, which are huge, suffer a strong cancellation. Actually, for sufficiently large energy, both the $S T$ and $K T$ terms are much larger than the unitarity bound $|\operatorname{Re} t| \leq s^{1 / 2} / 2 k \sim 1$, which is only satisfied by the real part of the total amplitude after their strong cancellation. In contrast, GKPY eqs. are dominated by $K T$ terms, which are always smaller than one. Remarkably, the $D T$ are still relatively small compared with the $K T$ despite there is one less subtraction in GKPY eqs. This means that the effect of high energy input, whose knowledge is less detailed, is well under control.

The error bands depicted in Figs. 1 and 2 are generated using a Monte Carlo Gaussian sampling of all parameters of the Constrained Fit to Data, (varied within 3 standard deviations). Uncertainties are just the widths of independent Gaussian fits to the left and right sides of the $S T, K T$ and $D T$ distributions. As seen in Fig. 1, for sufficiently high energies, the linear $s$ depen- dence of the $S T$ terms makes their uncertainty large and dominant when using RE for the $S 0$ and $S 2$ waves. This does not happen for GKPY eqs. whose errors, given the same input, are smaller, above $s \sim 400 \mathrm{MeV}$, than those from RE (see also Fig. 1 in J.R. Pelaez's talk in this conference). For this reason we are now implementing the GKPY eqs. together with FDR and standard Roy. eqs. not only to constraint the amplitude above $\sim 400 \mathrm{MeV}$, but also to extend analytically the amplitudes to determine the $\sigma$ pole position.

\section{Position of the $\sigma$ pole}

The mass and width of the $\sigma$ or $f_{0}(600)$ meson quoted in the Particle Data Table are very widely spread 9]

$M_{\sigma}-i \frac{\Gamma_{\sigma}}{2} \approx(400-1200)-i(250-500) \mathrm{MeV} .(2)$

The main reason of these uncertainties is that $\pi \pi$ scattering data are few and sometimes contradictory. Moreover, all the quoted theoretical models are not equally reliable, and even less so when extending the amplitude to the complex plane. Thus the position of the sigma pole in various models differ significantly [9], although, with a couple of exceptions, they tend to agree around $M_{\sigma}-i \Gamma_{\sigma} / 2 \approx(400-500)-i(220-300) \mathrm{MeV}$.

The recent data from E865 collaboration at Brookhaven [10] and from NA48/2 [11] provide us with new and very precise information on the $\pi \pi$ scattering at low energies. Thanks to these new data we are able to construct, with our Constrained Fits to Data, a very reliable description for the $S 0$ wave especially near the $\pi \pi$ threshold (see J.R. Peláez talk in this conference).

With those precise data parametrizations, we can now use either the Roy eqs. or the GKPY eqs. to extend the partial waves analytically to the complex plane and look for poles in the second sheet of the S-matrix. As it well known, a pole on the second Riemann sheet (unphysical sheet) is associated with a zero on the first - the physical one. Therefore, as usual, we look for zeroes of the physical sheet of the $S$-matrix,

$S_{0}^{0}(s)=1+2 i \sqrt{1-4 m_{\pi}^{2} / s} f_{0}^{0}(s)$. 
Depending on whether we use Roy or GKPY eqs. we find a different accuracy in our results, namely

$$
\begin{aligned}
& \sqrt{s_{\sigma}}=459_{-33}^{+36}-i 257_{-18}^{+17} \mathrm{MeV} \\
& \sqrt{s_{\sigma}}=461_{-15.5}^{+14.5}-i 255 \pm 16 \mathrm{MeV}
\end{aligned}
$$

from the GKPY equations. These values are in good agreement with each other. Note that the errors have been calculated in the same way as for the output amplitudes in Sect. 1, i.e. from a Monte Carlo Gaussian sampling. Except for those of the mass from RE, which are roughly $25 \%$ smaller, they are almost identical to the nonGaussian symmetric errors that we have been using in previous works 12

$\sqrt{s_{\sigma}}=(461 \pm 14)-i(255 \pm 16) \mathrm{MeV} \quad(\mathrm{GKPY})(6)$

It is reassuring that our error estimates remain stable calculating them in these two rather different ways.

This preliminary determination is quite consistent with other recent and precise results in the literature: On the one hand, both the mass and width lie less than 1.25 standard deviations from the prediction of twice-subtracted Roy's equations combined with ChPT results for the scattering lengths [8]: $\sqrt{s_{\sigma}}=441_{-8}^{+16}-i 272_{-14.5}^{+9} \mathrm{MeV}$. On the other hand our preliminary mass determination above is also slightly beyond one standard deviation from the pole in our simple fit 13. of a conformal expansion to low energy data $\sqrt{s_{\sigma}}=(484 \pm 17)-i(255 \pm 10) \mathrm{MeV}$, but agrees remarkably well on the width.

\section{Conclusions}

The GKPY equations - Roy-like dispersion relations with one subtraction for the $\pi \pi$ amplitudes - provide stringent constraints for dispersive analysis of experimental data. The main advantage of GKPY eqs. is that, for the same input, in the $0.4 \mathrm{GeV} \leq \sqrt{s} \leq 1.1 \mathrm{GeV}$ region they have significantly smaller errors than standard Roy. eqs. Hence, they provide better accuracy tests and analytic extensions of the amplitudes in that region. In particular, using just a data analysis consistent within errors with Forward Dispersion Relations, Roy eqs. and GKPY eqs. (and no
ChPT input), we have presented here the following preliminary but very precise determination of the $\sigma$ pole position:

$\sqrt{s_{\sigma}}=461_{-15.5}^{+14.5}-i 255 \pm 16 \mathrm{MeV}$

The details of GKPY eqs. together with a full constrained dispersive analysis the data, extending both GKPY and Roy eqs. up to $1.1 \mathrm{GeV}$ and some slight improvements on the partial waves is about to be finished. A final number for the $\sigma$ pole should follow relatively easy once that data analysis is completed.

\section{Acknowledgments}

This talk is dedicated to the memory of F.J. Yndurain. Partial financial support from Spanish contracts PR27/05-13955-BSCH, FPA200402602, UCM-CAM 910309 and BFM2003-00856.

\section{REFERENCES}

1. S.M. Roy, Phys. Lett. B 36, 353 (1971).

2. B. Ananthanarayan et al., Phys. Rept. 353, 207 (2001).

3. G. Colangelo, J. Gasser and H. Leutwyler, Nucl. Phys. B 603, 125 (2001).

4. S. Descotes-Genon, N. H. Fuchs, L. Girlanda and J. Stern, Eur. Phys. J. C 24, 469 (2002)

5. R. Kamiński, J.R. Pelaez and F.J. Yndurain, Phys. Rev. D 77, 054015 (2008).

6. R. Kaminski, J.R. Pelaez and F.J. Yndurain, Phys. Rev. D 74, 014001 (2006).

7. R. Kaminski, L. Lesniak and B. Loiseau, Phys. Lett. B 551, 241 (2003)

8. I. Caprini, G. Colangelo and H. Leutwyler, Phys. Rev. Lett. 96, 132001 (2006).

9. W. M. Yao et al., J. Phys. G33, 1-1232 (2006).

10. S. Pislak et al., Phys. Rev. Lett. 87, 221801 (2001).

11. J. R. Batley et al., CERN-PH-EP-2007-035 (2008).

12. R. Garcia-Martin et al., To appear in the proceedings of the Meson08 conference, Cracow, Poland.

13. F. J. Yndurain, R. Garcia-Martin and J. R. Pelaez, Phys. Rev. D 76, 074034 (2007) 

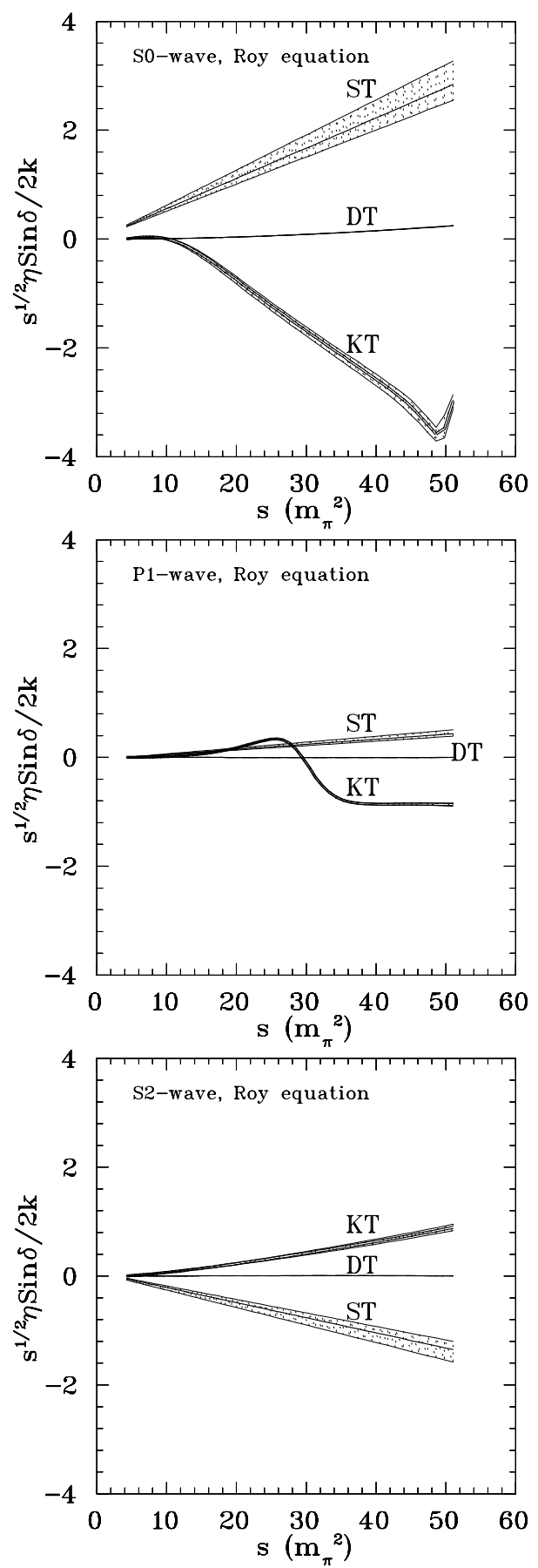

Figure 1. Subtraction $(S T)$, kernel $(K T)$ and driving $(D T)$ terms for the $S 0, P$ and $S 2$ waves from the twice-subtracted Roy's equations. Dashed bands denote the errors of these terms.
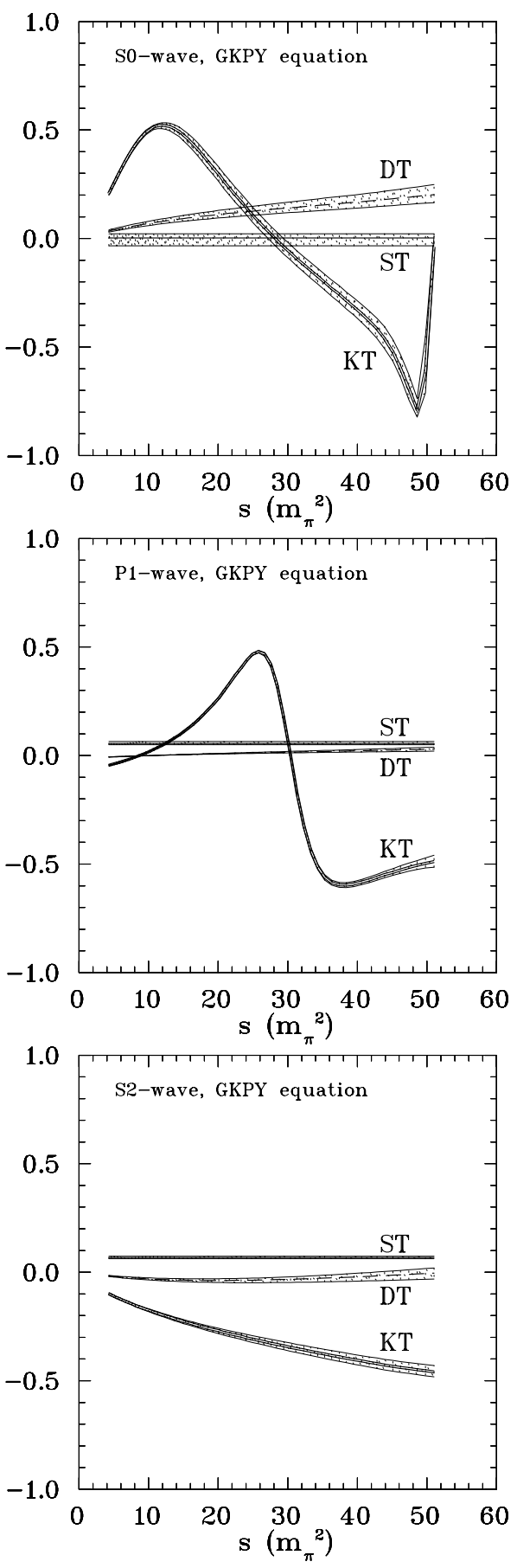

Figure 2. As in Fig. 1 but for GKPY equations. 